IASSNS-HEP-97/96

September, 1997

\title{
Gauge Fixing in the Partition Function for Generalized Quantum Dynamics
}

\author{
Stephen L. Adler \\ Institute for Advanced Study \\ Princeton, NJ 08540
}

Send correspondence to:

Stephen L. Adler

Institute for Advanced Study

Olden Lane, Princeton, NJ 08540

Phone 609-734-8051; FAX 609-924-8399; email adler@ias. edu 


\begin{abstract}
We discuss the problem of gauge fixing for the partition function in generalized quantum (or trace) dynamics, deriving analogs of the De WittFaddeev-Popov procedure and of the BRST invariance familiar in the functional integral context.
\end{abstract}




\section{Introduction, and the Gauge Theory Axial Gauge Partition Function}

Generalized quantum (or trace) dynamics ${ }^{1}$ is a generalization of classical mechanics, in which the Lagrangian and Hamiltonian are constructed as the trace of multinomials in noncommutative operator or matrix variables. This new form of analytic mechanics has a number of interesting properties. When the fermions are realized in the conventional manner as Grassmann matrices, generalized quantum dynamics incorporates and generalizes all rigid supersymmetry theories, such as ${ }^{2}$ the Wess-Zumino and supersymmetric Yang-Mills theories, and also $^{3}$ the recently much discussed "matrix model for M theory". In addition, one can formulate a statistical mechanics for generalized quantum dynamics, in both the canonical ${ }^{4}$ and microcanonical ${ }^{5}$ ensembles. Recent work suggests ${ }^{4,6}$ that this statistical mechanics can behave as a prequantum mechanics, with the Heisenberg commutation relations holding for statistical averages over the canonical ensemble of the underlying operator variables.

This prior work on the statistical mechanics of generalized quantum dynamics has assumed either that one is dealing with an unconstrained system, or that the constraints have been explicitly integrated out. In the generic case for an unconstrained system, the canonical ensemble takes the form ${ }^{4,5,6}$

$$
\begin{aligned}
& \rho=Z^{-1} \exp (-\tau \mathbf{H}-\operatorname{Tr} \tilde{\lambda} \tilde{C}), \\
& Z=\int d \mu \exp (-\tau \mathbf{H}-\operatorname{Tr} \tilde{\lambda} \tilde{C}),
\end{aligned}
$$

with $d \mu$ the invariant matrix (or operator) phase space measure provided ${ }^{4}$ by Liouville's

theorem, with $\mathbf{H}$ the conserved total trace Hamiltonian, and with $\tilde{C}$ the conserved operator

$$
\tilde{C}=\sum_{r}\left[q_{r}, p_{r}\right]-\sum_{r \text { fesons }}\left\{q_{r}, p_{r}\right\}
$$


In order to apply Eq. (1a) directly to a constrained system, one must first explicitly integrate out the constraints. A simple example where this is possible, that forms the focus of this paper, is provided by the generalized quantum dynamics form of non-Abelian gauge theory, ${ }^{1}$ which has a full operator valued gauge invariance group. Let $A_{\nu}$ be an anti-selfadjoint operator or matrix valued gauge potential, let $F_{\mu \nu}$ be the corresponding gauge field strength defined by

$$
F_{\mu \nu}=\partial_{\mu} A_{\nu}-\partial_{\nu} A_{\mu}+\left[A_{\mu}, A_{\nu}\right]
$$

and let $\mathbf{L}$ be the total trace Lagrangian defined by

$$
\mathbf{L}=\frac{1}{4} \operatorname{Tr} F_{\mu \nu} F^{\mu \nu}
$$

As is well known, Eqs. (2a, b) define a constrained dynamical system. Introducing the canonical momenta

$$
p_{A_{\ell}}=-F_{0 \ell}, \quad \ell=1,2,3
$$

and the covariant derivative

$$
D_{\mu} X=\partial_{\mu} X+\left[A_{\mu}, X\right]
$$

the constraint equation for the system of Eqs. (2a, b) takes the form

$$
\sum_{\ell=1}^{3} D_{\ell} p_{A_{\ell}}=0
$$

Since in axial gauge, where $A_{3}=0$, the covariant derivative $D_{3}$ simplifies to $D_{3}=\partial_{3}$, the constraint of Eq. (3c) is readily integrated out, giving as the explicit expression for the total 
trace Hamiltonian,

$$
\begin{aligned}
\mathbf{H} & =\operatorname{Tr}\left[-\frac{1}{2} \sum_{\ell=1}^{3} p_{A_{\ell}}^{2}-\frac{1}{4} \sum_{\ell, m=1}^{3} F_{\ell m}^{2}\right] \\
& =\int d^{3} x \frac{1}{2} \operatorname{Tr}\left[-\sum_{\ell=1}^{2} p_{A_{\ell}}^{2}-F_{03}^{2}-F_{12}^{2}-\sum_{\ell=1}^{2}\left(\partial_{3} A_{\ell}\right)^{2}\right],
\end{aligned}
$$

with $F_{03}$ given by the line integral

$$
F_{03}=\left.\int d z^{\prime} \frac{1}{2} \frac{z-z^{\prime}}{\left|z-z^{\prime}\right|}\left(\sum_{\ell=1}^{2} D_{\ell} p_{A_{\ell}}\right)\right|_{z^{\prime}}
$$

The conserved operator $\tilde{C}$ takes the form, in a general gauge,

$$
\tilde{C}=\int d^{3} x \sum_{\ell=1}^{3}\left[A_{\ell}, p_{A_{\ell}}\right]=\sum_{\ell=1}^{3}\left(\int d^{3} x D_{\ell} p_{A_{\ell}}-\int d S_{\ell} p_{A_{\ell}}\right)
$$

with $d S_{\ell}$ the surface element of the sphere at infinity. To specialize Eq. (5a) to axial gauge, one sets $A_{3}$ equal to zero and evaluates $p_{A_{3}}$ using Eqs. (3a) and (4b). The axial gauge partition function is then given by Eq. (1a), with the phase space measure $d \mu$ given by

$$
d \mu_{\mathrm{axial}}=\prod_{\vec{x}} \prod_{\ell=1}^{2} d A_{\ell}(\vec{x}) d p_{A_{\ell}(\vec{x})}
$$

The problem we wish to address in this paper is how to generalize the axial gauge partition function to other gauges in which it may not be possible to explicitly integrate out the constraint. This is a familiar problem in the theory of path integrals, and we shall use methods similar to the ones employed there to give a solution. However, since the partition function singles out a Lorentz frame, we will have to make a restriction not encountered in the Lorentz scalar path integral case, namely we will consider only nontemporal gauge conditions that do not involve the scalar potential $A_{0}$. This will still allow us to consider gauge transformations that rotate the axial gauge axis, or that transform to rotationally invariant gauges such as Coulomb gauge. We will also make the further assumption that 
the allowed gauge transformations leave invariant the surface integral contribution to $\operatorname{Tr} \tilde{\lambda} \tilde{C}$ arising from the second term on the right hand side of Eq. (5a), which places a restriction on the constant of integration that governs the gauge transformation at the point at infinity. [Although the volume integral in the first term on the right hand side of Eq. (5a) is generically gauge covariant rather than gauge invariant, this causes no problem because its integrand is proportional to the constraint of Eq. (3c).] In Sec. 2 we develop an analog of the standard DeWitt-Faddeev-Popov method to write the axial gauge partition function in a general nontemporal gauge, subject to the surface term restriction just stated. Since we have shown $^{2,3}$ that trace dynamics incorporates rigid supersymmetry, and since BRST invariance is a particular rigid supersymmetry transformation, it is not surprising that the generalized expression for the partition function, when reexpressed in terms of ghost fermions, admits a BRST invariance, and this is demonstrated in Sec. 3. In the analysis that follows, we do not attempt to address the issue of convergence of the partition function, which may well require significant restrictions on the class of trace Hamiltonians being considered.

\section{General Nontemporal Gauges and the De Witt-Faddeev-Popov Procedure}

To express the partition function in a general nontemporal gauge, we follow closely the treatment of the De Witt-Faddeev-Popov construction in the familiar functional integral case, as given in the text of Weinberg ${ }^{7}$. Let us consider the integral

$$
\begin{aligned}
Z_{G} & =\int d \mu B\left[f\left(A_{\ell}\right)\right] \delta(D) \operatorname{det} \mathcal{F}\left[A_{\ell}\right] \exp (-\tau \mathbf{H}-\operatorname{Tr} \tilde{\lambda} \tilde{C}), \\
d \mu & =\prod_{\vec{x}} \prod_{\ell=1}^{3} d A_{\ell}(\vec{x}) d p_{A_{\ell}(\vec{x})},
\end{aligned}
$$

with $\mathbf{H}$ given by the first line of Eq. (4a) [which is valid in a general gauge on the constraint 
surface $D=0$ selected by the delta function in Eq. (6a)], with $\tilde{C}$ given by Eq. (5a), and with $D$ defined by $D \equiv \sum_{\ell=1}^{3} D_{\ell} p_{A_{\ell}}$. The delta function of the anti-self-adjoint matrix valued argument $D$ appearing in Eq. (6a) is given, in terms of ordinary delta functions of the real $(R)$ and imaginary $(I)$ parts of the matrix elements, by

$$
\delta(D)=\prod_{m<n} \delta\left(\left(D_{R}\right)_{m n}\right) \prod_{m \leq n} \delta\left(\left(D_{I}\right)_{m n}\right)
$$

and the integration measure over the anti-self-adjoint matrix $A_{\ell}$ is defined by

$$
d A_{\ell}=\prod_{m<n} d\left(A_{\ell R}\right)_{m n} \prod_{m \leq n} d\left(A_{\ell I}\right)_{m n}
$$

and similarly for $d p_{A_{\ell}}$. The function $B[f]$ is an arbitrary integrable scalar valued function of the matrix valued argument $f\left(A_{\ell}\right)$, which is used to specify the gauge condition. We shall treat $f$ as a column vector $f_{\alpha}$ with $\alpha$ a composite index formed from the matrix row and column indices $m, n$; the argument $\mathcal{F}$ of the De Witt-Faddeev-Popov determinant is then given in terms of $f$ by the expression

$$
\left.\mathcal{F}_{\alpha \vec{x}, \beta \vec{y}}\left[A_{\ell}\right] \equiv \frac{\delta f_{\alpha}\left(A_{\ell}(\vec{x})+D_{\ell} \Lambda(\vec{x})\right)}{\delta \Lambda_{\beta}(\vec{y})}\right|_{\Lambda=0}
$$

where $\delta$ is the usual functional derivative and $\beta$ is the composite of the row and column indices of the infinitesimal gauge transformation matrix $\Lambda$.

We now demonstrate two properties of the integral $Z_{G}$ defined in Eq. (6a): (i) first, we show that when the gauge fixing functions $B[f]$ and $f\left(A_{\ell}\right)$ are chosen to correspond to the axial gauge condition, then Eq. (6a) reduces (up to an overall constant) to the axial gauge partition function of Sec. 1; (ii) second, we show that $Z_{G}$ is in fact independent of the function $f\left(A_{\ell}\right)$, and depends on the function $B[f]$ only through an overall constant. These two properties together imply that $Z_{G}$ gives the wanted extension of the axial gauge partition function to general nontemporal gauges. 
To establish property (i), we make the conventional axial gauge choice

$$
B\left[f\left(A_{\ell}\right)\right]=\delta\left(A_{3}\right)=\prod_{m<n} \delta\left(\left(A_{3 R}\right)_{m n}\right) \prod_{m \leq n} \delta\left(\left(A_{3 I}\right)_{m n}\right)
$$

so that

$$
\int d A_{3} B\left[f\left(A_{\ell}\right)\right]=\int d A_{3} \delta\left(A_{3}\right)=1
$$

With this gauge choice,

$$
D_{3} p_{A_{3}}=\partial_{3} p_{A_{3}}
$$

which implies that

$$
\begin{aligned}
\delta(D) & =\delta\left(\partial_{3} p_{A_{3}}+\sum_{\ell=1}^{2} D_{\ell} p_{A_{\ell}}\right) \\
& =\left|\partial_{3}\right|^{-1} \delta\left(p_{A_{3}}+\int^{z} d z^{\prime} \sum_{\ell=1}^{2} D_{\ell} p_{A_{\ell}}\right) ;
\end{aligned}
$$

hence the integral over $p_{A_{3}}$ in $Z_{G}$ can be done explicitly, giving (up to an overall constant factor coming from the Jacobian $\left|\partial_{3}\right|^{-1}$ ) the expression

$$
Z_{G}=\left.\int d \mu_{\text {axial }} \exp (-\tau \mathbf{H}-\operatorname{Tr} \tilde{\lambda} \tilde{C})\right|_{A_{3}=0 ; p_{A_{3}}=-\int^{z} d z^{\prime} \sum_{\ell=1}^{2} D_{\ell} p_{A_{\ell}}}
$$

which agrees with the axial gauge partition function of Sec. 1.

To establish property (ii), we first examine the gauge transformation properties of the various factors in the integral defining $Z_{G}$. We begin with the integration measure $d \mu$. Under the infinitesimal gauge transformation (with $\Lambda$ anti-self-adjoint)

$$
A_{\ell} \rightarrow A_{\ell}+D_{\ell} \Lambda=A_{\ell}+\partial_{\ell} \Lambda+\left[A_{\ell}, \Lambda\right]
$$

the inhomogeneous term $\partial_{\ell} \Lambda$ does not contribute to the transformation of the differential $d A_{\ell}$, and so $d A_{\ell}$ obeys the homogeneous transformation law $d A_{\ell} \rightarrow d A_{\ell}+\Delta_{\ell}$, with

$$
\Delta_{\ell} \equiv\left[d A_{\ell}, \Lambda\right]
$$


Hence to first order in $\Lambda$, the Jacobian of the transformation is

$$
\begin{aligned}
J & =1+\sum_{m<n} \frac{\partial\left(\Delta_{\ell R}\right)_{m n}}{\partial\left(d A_{\ell R}\right)_{m n}}+\sum_{m \leq n} \frac{\partial\left(\Delta_{\ell I}\right)_{m n}}{\partial\left(d A_{\ell I}\right)_{m n}} \\
& =1+\left(\sum_{m<n}+\sum_{m \leq n}\right)\left[\left(\Lambda_{R}\right)_{n n}-\left(\Lambda_{R}\right)_{m m}\right] \\
& =1 \quad,
\end{aligned}
$$

since the anti-self-adjointness of $\Lambda$ implies that $\left(\Lambda_{R}\right)_{n m}=-\left(\Lambda_{R}\right)_{m n}$, and so the diagonal matrix elements $\left(\Lambda_{R}\right)_{n n}$ are all zero. Thus each factor $d A_{\ell}(\vec{x})$ in the integration measure is gauge invariant. A similar argument applies to each factor $d p_{A_{\ell}(\vec{x})}$ in the integration measure, and also to the factor $\delta(D)$ in the integrand, since $D$ obeys the homogeneous gauge transformation law $D \rightarrow D+[D, \Lambda]$. Turning to the exponential, the terms $\operatorname{Tr} p_{A_{\ell}}^{2}$ and $\operatorname{Tr} F_{\ell m}^{2}$ are gauge invariant, and so the trace Hamiltonian $\mathbf{H}$ is gauge invariant. From Eq. (5a), the volume integral term in $\tilde{C}$ vanishes by virtue of the integrand factor $\delta(D)$, and so $\operatorname{Tr} \tilde{\lambda} \tilde{C}$ receives a contribution only from the surface term in Eq. (5a), which by hypothesis is left invariant by the class of gauge transformations under consideration. In sum, we see that the integral $Z_{G}$ has the form

$$
Z_{G}=\int d \mu \mathcal{G}\left[A_{\ell}\right] B\left[f\left(A_{\ell}\right)\right] \operatorname{det} \mathcal{F}\left[A_{\ell}\right]
$$

with the integration measure $d \mu$ and the integrand factor

$$
\mathcal{G}\left[A_{\ell}\right]=\delta(D) \exp (-\tau \mathbf{H}-\operatorname{Tr} \tilde{\lambda} \tilde{C})
$$

both gauge invariant. Hence $Z_{G}$ has exactly the form assumed in the discussion of Weinberg ${ }^{7}$, and the proof given there completes the demonstration of property (ii). 


\section{Ghosts and BRST Invariance of the Generalized Partition Function}

Let us continue to follow the standard path integral analysis, and represent the De Witt-Faddeev-Popov determinant $\operatorname{det} \mathcal{F}\left[A_{\ell}\right]$ as an integral over fermionic ghosts, by writing

$$
\operatorname{det} \mathcal{F}\left[A_{\ell}\right]=\int d \omega^{*} d \omega \exp \left(\int d^{3} x d^{3} y \omega_{\alpha}^{*}(\vec{x}) \mathcal{F}_{\alpha \vec{x}, \beta \vec{y}}\left[A_{\ell}\right] \omega_{\beta}(\vec{y})\right)
$$

with

$$
d \omega=\prod_{\vec{x}} \prod_{m, n} d \omega_{m n}(\vec{x}) \quad, \quad d \omega^{*}=\prod_{\vec{x}} \prod_{m, n} d \omega_{m n}^{*}(\vec{x})
$$

Let us also take for $B[f]$ the usual Gaussian

$$
B[f]=\exp \left(-\frac{1}{2 \xi} \int d^{3} x \operatorname{Tr} f\left(A_{\ell}(\vec{x})\right)^{2}\right)
$$

and for $f\left(A_{\ell}\right)$ the linear gauge condition

$$
f\left(A_{\ell}\right)=\sum_{\ell} L^{\ell} A_{\ell}
$$

in which $L^{\ell}$ can be either a fixed vector (such as $\delta_{\ell 3}$ in axial gauge) or a differential operator (such as $\partial_{\ell}$ in Coulomb gauge), and a summation of $\ell$ from 1 to 3 is understood. With this choice of $f\left(A_{\ell}\right)$, we find from Eq. (6d) that

$$
\begin{aligned}
\mathcal{F}_{n m \vec{x}, p q \vec{y}}\left[A_{\ell}\right] & =\frac{\delta f_{n m}\left(A_{\ell}(\vec{x})+D_{\ell} \Lambda(\vec{x})\right)}{\delta \Lambda_{p q}(\vec{y})} \\
& =\sum_{\ell} L_{\vec{x}}^{\ell}\left(\frac{\partial \delta(\vec{x}-\vec{y})}{\partial x^{\ell}} \delta_{n p} \delta_{m q}+\delta(\vec{x}-\vec{y})\left[\left(A_{\ell}\right)_{n p} \delta_{m q}-\delta_{n p}\left(A_{\ell}\right)_{q m}\right]\right)
\end{aligned}
$$

which when substituted into the exponent in Eq. (11a) gives, after integrations by parts,

$$
\left.\int d^{3} x d^{3} y \omega_{\alpha}^{*}(\vec{x}) \mathcal{F}_{\alpha \vec{x}, \beta \vec{y}}\left[A_{\ell}\right] \omega_{\beta}(\vec{y})\right)=\int d^{3} x \operatorname{Tr} \bar{\omega}(\vec{x}) \sum_{\ell} L^{\ell} D_{\ell} \omega(\vec{x})
$$

where we have defined $\bar{\omega}_{m n}=\omega_{n m}^{*}$. Hence the expression of Eq. (10a) for $Z_{G}$ becomes

$$
Z_{G}=\int d \mu d \bar{\omega} d \omega \mathcal{G}\left[A_{\ell}\right] \exp \left[-\int d^{3} x \operatorname{Tr}\left(\frac{1}{2 \xi}\left(\sum_{\ell} L^{\ell} A_{\ell}\right)^{2}-\bar{\omega}(\vec{x}) \sum_{\ell} L^{\ell} D_{\ell} \omega(\vec{x})\right)\right]
$$


An alternative way of writing Eq. (13a), that is convenient for exhibiting the BRST invariance, is to introduce an auxilliary self-adjoint matrix field $h$ and to reexpress Eq. (13a) as

$$
\left.Z_{G}=\int d \mu d h d \bar{\omega} d \omega \mathcal{G}\left[A_{\ell}\right] \exp \left[-\int d^{3} x \operatorname{Tr}\left(\frac{\xi}{2} h^{2}+i h \sum_{\ell} L^{\ell} A_{\ell}\right)-\bar{\omega}(\vec{x}) \sum_{\ell} L^{\ell} D_{\ell} \omega(\vec{x})\right)\right]
$$

Starting from Eq. (13b), we can now show that $Z_{G}$ has a BRST invariance of the familiar form. Let $\theta$ be an $\vec{x}$-independent $c$-number Grassmann parameter (i.e., a $1 \times 1$ Grassmann matrix), and consider the variations defined by

$$
\begin{gathered}
\delta \omega=\omega^{2} \theta \\
\delta A_{\ell}=D_{\ell} \omega \theta \\
\delta \bar{\omega}=-i h \theta \\
\delta h=0 .
\end{gathered}
$$

We begin by showing that Eq. (14) defines a nilpotent transformation, in the sense that the second variations of all quantities are zero. To verify this, we show that the variations of $\omega^{2}$ and $D_{\ell} \omega$ are zero (the variations of $h$ and of 0 are trivially 0 ), as follows:

$$
\begin{aligned}
\delta \omega^{2} & =\{\delta \omega, \omega\}=\left\{\omega^{2} \theta, \omega\right\}=\omega^{2}\{\omega, \theta\}=0, \\
\delta D_{\ell} \omega & =\left[\delta A_{\ell}, \omega\right]+D_{\ell} \delta \omega=\left[D_{\ell} \omega \theta, \omega\right]+D_{\ell} \omega^{2} \theta=-\left\{D_{\ell} \omega, \omega\right\} \theta+\left\{D_{\ell} \omega, \omega\right\} \theta=0 .
\end{aligned}
$$

To see that $Z_{G}$ is invariant, we note that the action on $A_{\ell}$ of the BRST transformation of Eq. (14) is just a gauge transformation (albeit with a Grassmann valued parameter), and so the gauge invariance analysis of Sec. 2 shows that the factors $d \mu$ and $\mathcal{G}\left[A_{\ell}\right]$ are invariant. The measure $d h$ is trivially invariant, and the measure $d \bar{\omega}$ is invariant because $\delta \bar{\omega}$ has no dependence on $\bar{\omega}$. Since

$$
\delta(d \omega)=d(\delta \omega)=d\left(\omega^{2} \theta\right)=(\omega d \omega+d \omega \omega) \theta,
$$


we have

$$
(\delta(d \omega))_{m n}=\left(\omega_{m m} d \omega_{m n}+d \omega_{m n} \omega_{n n}\right) \theta+\ldots=d \omega_{m n}\left(\omega_{n n}-\omega_{m m}\right) \theta+\ldots
$$

with ... denoting terms that contain matrix elements $d \omega_{m^{\prime} n^{\prime}}$ with $\left(m^{\prime}, n^{\prime}\right) \neq(m, n)$. Hence the Jacobian of transformation for $d \omega$ differs from unity by a term proportional to

$$
\sum_{n m}\left(\omega_{n n}-\omega_{m m}\right) \theta=0
$$

and so the measure $d \omega$ is also invariant. Hence to complete the demonstration that $Z_{G}$ is BRST invariant, we have to show that the gauge fixing part of the Hamiltonian,

$$
\mathbf{H}_{G} \equiv \int d^{3} x \operatorname{Tr}\left(\frac{\xi}{2} h^{2}+i h \sum_{\ell} L^{\ell} A_{\ell}-\bar{\omega} \sum_{\ell} L^{\ell} D_{\ell} \omega\right)
$$

is BRST invariant. Since we have already seen that $D_{\ell} \omega$ is invariant, and since $h$ is trivially invariant, we have only to verify that

$$
0=\int d^{3} x \operatorname{Tr}\left[i h \sum_{\ell} L^{\ell} \delta A_{\ell}-(\delta \bar{\omega}) \sum_{\ell} L^{\ell} D_{\ell} \omega\right]=\int d^{3} x \operatorname{Tr} i h \sum_{\ell} L^{\ell} D_{\ell}\{\omega, \theta\}
$$

which checks, completing the demonstration of BRST invariance of the generalized partition function.

\section{Acknowledgments}

I wish to thank Lowell Brown for helpful conversations, and to acknowledge the hospitality of the Aspen Center for Physics, where this work was done. This work was supported in part by the Department of Energy under Grant \#DE-FG02-90ER40542. 


\section{References}

[1] S. L. Adler, Nucl. Phys. B415, 195 (1994); S. L. Adler, Quaternionic Quantum Mechanics and Quantum Fields, Secs. 13.5-13.7 (Oxford U. P., New York, 1995).

[2] S. L. Adler, preprint IASSNS-HEP-97/16, hep-th/9703132, Nucl. Phys. B (in press).

[3] S. L. Adler, preprint IASSNS-HEP-97/15, hep-th/9703053, Phys. Lett. B (in press).

[4] S. L. Adler and A. C. Millard, Nucl Phys. B473, 199 (1996).

[5] S. L. Adler and L. P. Horwitz, J. Math. Phys. 37, 5429 (1996).

[6] S. L. Adler and A. Kempf, preprint IASSNS-HEP-97/51, DAMTP-97-99, hep-th 9709106 .

[7] S. Weinberg, The Quantum Theory of Fields, Vol. II, Sec. 15.5 (Cambridge U. P., Cambridge, 1996.) 